\title{
Implementing security at the external interfaces of TMNs
}

\author{
George Andrianopoulos, ATC Bull (andriano@01p.gr) \\ Nicolas Ganivet, Alcatel-ISR (ganivet@isr.alcatel-alsthom.fr) \\ Lars Hofseth,Telscom (hofseth@vptt.ch) \\ Dominique Maillot,Telis (Maillot@sqy.tel.telis.fr) \\ Jon Ølnes, Norwegian Computing Centre, (Jon.Olnes@nr.no) \\ Linda Strick, GMD FOKUS, (strick@fokus.gmd.de)
}

\begin{abstract}
This paper presents the implementation of security solutions for securing the X-interfaces between Telecommunication Management Networks (TMN) as specified by the ACTS project TRUMPET (ACTS112-D2, 1996) (ACTS112-D7, 1997). The basis for the implementation specifications is the TRUMPET security architecture which identifies the main components and their interfaces, and their integration with the TMN platform. Following a definition of the foundations and framework to use for the specifications, establishment of a secure management association is specified. This consists of security context establishment and peerentity authentication. The security context selects the security services and mechanisms to use for the association, and their parameters such as cryptographic keys. Then, per management message, security services are specified as additional objects and interfaces between them. Non-repudiation and the support services key management and security audit and alarm, although encompassed by TRUMPET, are not detailed in this paper.
\end{abstract}

\section{Keywords}

Telecommunications management, TMN, external interface, security, integrity, confidentiality. 


\section{INTRODUCTION}

This article presents the implementation specifications of the components that constitute TRUMPET's package for securing inter-domain management between TMN (ITU-T M.3010, 1995 ) systems. The steps taken in TRUMPET to specify the security solutions are :

$\lambda$ Describe security policies, defined as selections of security services and mechanisms and their placement with respect to the communication protocol stack, to apply for interdomain management. Several policies are needed to meet requirements of varying severity.

$\lambda$ Describe a system architecture, i.e. components and interfaces, suitable for implementation of the security policies within a TMN Operations System (OS) acting either as manager or agent. Integration with the TMN architecture, in particular the communication protocols, is crucial. Architectures for open protocol stacks (i.e. the internals of the stack are accessible for modifications) and closed stacks (i.e. security can only be added on top of the OSI stack) are described.

$\lambda$ Detail this architecture in terms of specifications of the components and their interfaces.

Only the closed OSI stack architecture is further elaborated, since TRUMPET's reference

TMN platform is a commercial one.

Section 2 describes the foundations and framework used in the specifications, mainly based on concepts from CORBA and TINA-C.

Section 3 gives an overview of the overall system architecture in terms of components and the interfaces between these.

The way these components and interfaces are actually used for a particular management association is determined through the establishment of a security context at the time of association establishment. This is described in Section 4, which also handles the peer-entity authentication service. A security context consists of a selection of security services and mechanisms, and necessary parameters, like cryptographic keys.

In the following sections, specifications are given for the individual services access control, data integrity and confidentiality. Key management, security audit and alarm, non-repudiation and management of security are specified by TRUMPET but not detailed in this paper.

\section{FOUNDATIONS AND FRAMEWORK}

\subsection{Definitions}

TRUMPET's specification of the security components uses concepts from (CORBA, 1994) and TINA-C. TINA ODL (object definition language) is used as a specification language, because it allows definition of relationships encompassing multiple interfaces. Nevertheless, interface specifications and operation signatures are the same as for CORBA IDL (Interface Description Language).

\section{Component}

A component is a functionality or set of functionalities which is meant to be used by other components. Components represent a high level of abstraction, encapsulating the component 
internals from the environment. Components may consist of one or more objects and internal interfaces between these objects. The interfaces which are visible externally to the component, are called contracts and can be used by other components. Thus, components can be seen as a composition of fine-grained objects with well defined external behaviour at their contracts. Components are designed and packed to be reusable.

In the specification, the interfaces are indicated as a simple list of optionally scoped identifiers. A graphical notation for components is shown in Figure 1 (a).

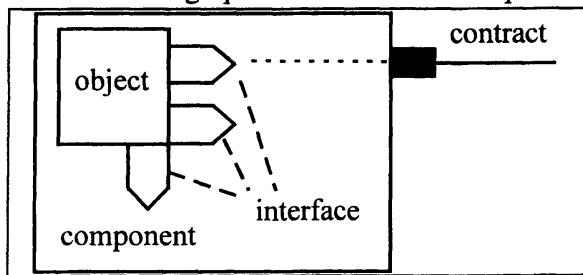

(a)

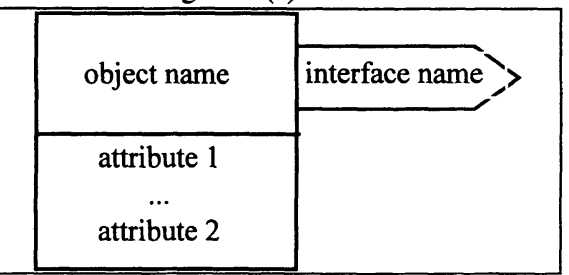

(b)

Figure 1 Graphical notation for (a) components (b) objects and interfaces

\section{Object and interface}

Templates are defined for objects and interfaces and are here only summarised. A graphical notation for object class and interface is shown in Figure 1 (b).

An object is an identifiable, encapsulated entity that provides one or more interfaces that can be requested by a client. An interface is a description of a set of possible operations that a client may request of an object An operation is an identifiable entity that denotes a service that can be requested. An operation has a signature that describes the values of requested parameters and returned results. Additionally an operation should have specification of exceptions, specification of contextual information that may affect the request for the operation. Parameter, exceptions and attributes are used as defined by CORBA-IDL.

\subsection{Specification steps}

To derive a specification, the following steps are taken :

1. breakdown the security service into components;

2. specify the components;

3. describe the interactions between objects.

The components are identified according to the level of functionality which will be provided to other components in order to fulfil a required task. They are specified in detail by using object and interface templates as discussed above. To show the behaviour of interactions and to avoid unintended effects of a object behaviour, it may be necessary to describe some interactions between objects or components using ETDs (Event Trace Diagram) (Rumbaugh, 1991). 


\section{SECURITY ARCHITECTURE OVERVIEW}

The security architecture consists of a set of security components, which can be used by TMN platforms with open or closed protocol stacks. The distinction is described as :

$\lambda$ For an open platform, typically a research management platform, the internals of the protocol stack can be accessed for additions and modifications. In this case, TRUMPET suggests use of the Generic Upper Layer Security (GULS) specifications (ITU-T X.830833,1995 ) and the Transport Layer Security Protocol (TLSP) (ITU-T X.274, 1993).

$\lambda$ For a closed platform, typically a commercial management platform, security features can only be added on top of the interface to the protocol stack, i.e. over CMISE, ACSE etc.

Since TRUMPET has selected a commercial TMN platform for implementation, only the commercial platform architecture has been further developed into component specifications, and is presented here. Data integrity, confidentiality and non-repudiation are especially difficult to implement if the protocol stack is not accessible, as discussed later in this paper.

TRUMPET's security policies prescribe the use of public key mechanisms for authentication, non-repudiation, and possibly also for key management (of secret keys). Use of Trusted Third Parties (TTP) in the role of Certification Authorities (CA) is anticipated. Symmetric encryption is used for confidentiality and data integrity protection.

The communication to secure is between a Management Application Entity (MAE) belonging to an OS in one domain to another MAE belonging to an OS in another domain. Different MAEs within an OS may use different security profiles, and the choice of security profile is made during the initialisation of the security context. Selection of a security profile may be constrained by the mechanisms supported by an implementation, by internal policies, or by target OS policies. The architecture must support all security profiles because it is not a priori possible to determine which security policies that will be applied to a particular $\mathrm{X}$ interface. However, some policy decisions inherently effects the architecture, like the decision to use public key cryptography.

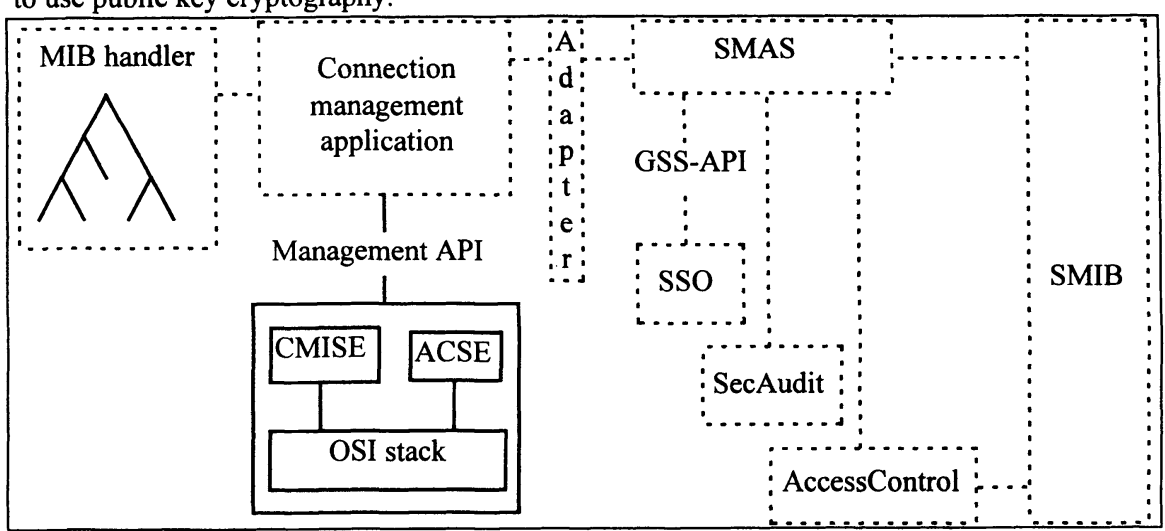

Figure 2 Architecture for a commercial management platform.

When a MAE belonging to the initiator OS performs inter-domain management operations, it may be working on behalf of another entity (a human user or another MAE) or on its own 
behalf. To preserve privacy of users, and to facilitate management of access privileges (authorisation), the MAE will always use its own identity and associated set of privileges to perform the management operations on the target OS. This implies that proper internal security measures must be enforced before human operators or MAEs are given access to the management capabilities of MAEs that perform inter-domain management.

To be as independent as possible from the management application and its environments, the security components are accessed through an Adapter component. Ideally, the interfaces provided by the Adapter to the application should be identical to the interfaces the application uses for access to the communication services. Alternatively, a defined interface (like the GSS-API, see below) could be offered from the security components, in which case the applications must incorporate this interface, and perform necessary transformations on the data passed over the interface.

To achieve a high degree of flexibility to particular security mechanisms, the architecture is based on the Generic Security Service API (GSS-API) (RFC 1508, 1993). The GSS-API interfaces to a Security Support Object (SSO) used to establish a security context between the communication parties and to perform security transformations on the application data.

With respect to a commercial management platform, there is some minimal support required for the transfer of security data between communicating parties. The specific requirements that must be satisfied are :

$\lambda$ the authentication field of ACSE must be supported to establish the security context and for authentication;

$\lambda$ the access control field of CMIS management operations must be supported to transfer security related information.

The security architecture also requires that agents have control over accesses to the MIB. This is necessary to enforce access control to MOs. Although TRUMPET is responsible for the implementation of agents, their design may be restricted by tools provided by the platform provider. For example, the code generated by a GDMO compiler may not be compatible with the introduction of access control mechanisms.

The architecture shown in Figure 2 is able to support most of the security services required by the TRUMPET policies. The security services that cannot be fully supported are integrity, confidentiality, non-repudiation and security negotiations.

When encryption for confidentiality is performed above CMISE, the encrypted data must be inserted into one of the fields of the particular CMIS operation being requested. However, most of the fields have specific pre-defined types that cannot accommodate an encrypted data type. In general, the only exception, and the only fields that can be encrypted, are the fields used to carry the attribute values to and from the target MIB.

\section{SECURE MANAGEMENT ASSOCIATION ESTABLISHMENT}

\subsection{Secure Management Association Support component}

The Secure Management Association Support component (SMASC) is that component of the management system which provides the management applications with the means to secure 
associations with other management applications located in another management system. The main purpose of the $S M A S C$ is to isolate the security-related components from the application code. This approach has the following advantages :

$\lambda$ security can be added to an existing application, without effecting its internal structure;

$\lambda$ the security-related code can be designed, programmed and verified independently;

$\lambda$ addition of auditing capabilities for security-related events is made easier and safer;

$\lambda$ the resulting code can easily be customized to accommodate new security policies.

Figure 3 shows the main components of the security architecture, the internal structure of the $S M A S C$, and the contract interfaces of the $S M A S C$ to other components. The security services of the $S M A S C$ are accessed through an Adapter Component whose purpose is to transform platform specific syntax (e.g. XOM objects (XOM, 1991)) to generic data structures (e.g. BER encoding). Thus, platform specific code can be restricted to the Adapter component, and the $S M A S C$ can be reused in different environments without major modifications.

The $S M A S C$ is also interfaced with :

$\lambda$ the AccessControl component to perform access control to management associations, and access control to operations on managed objects;

$\lambda$ the Event and Alarm component to log relevant security events occurring on the management associations - this component interfaces to further components for auditing.

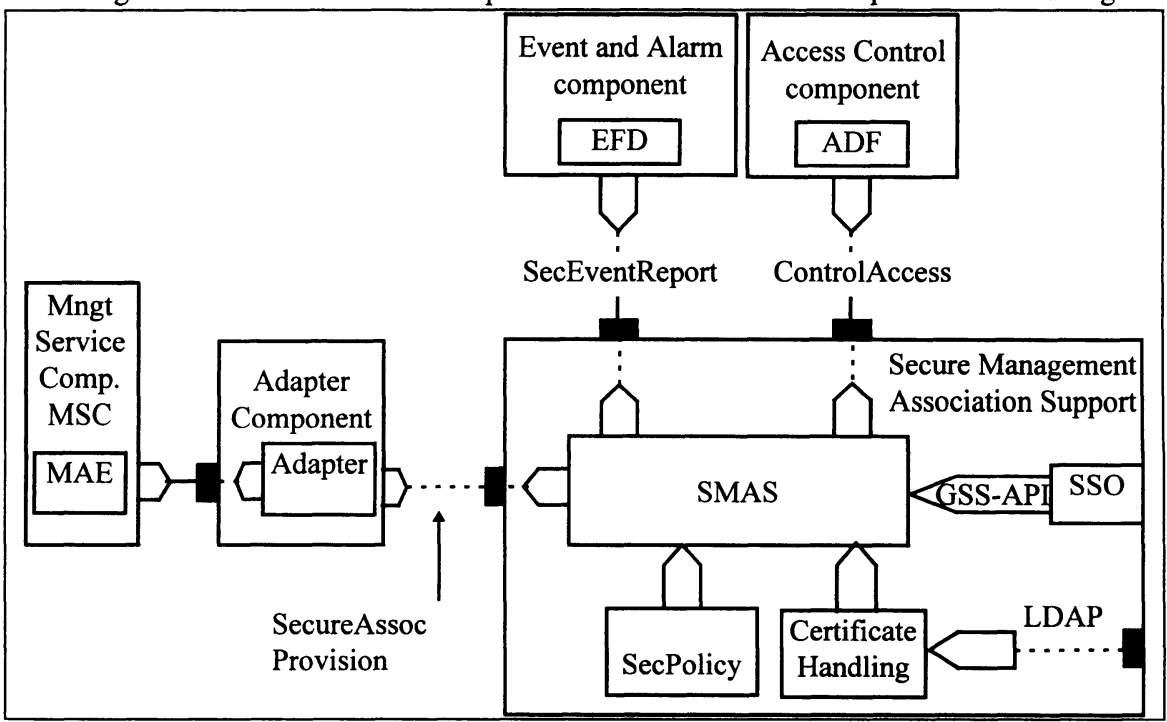

Figure 3 Graphical representation of the SMAS component.

The SMASC component is decomposed into object classes as follows :

$\lambda$ a Secure Management Association Support object, $S M A S$, which co-ordinates the behavior of the whole component;

$\lambda$ a System Security Object, $S S O$, which is responsible for negotiating and storing the security context, and for provisioning of generic security services such as authentication, 
integrity, encryption and digital signatures, via an interface compatible to the Generic Security Service Application Program Interface (GSS-API) (RFC 1508, 1993) (GSS V2, 1996);

$\lambda$ a SecPolicy object which contains the policy rules for interaction with a remote MAE;

$\lambda$ a CertificateHandling object, which performs key handling such as caching, fetching certificates from directories and checking Certificate Revocation Lists (CRL).

The SSO may be implemented using existing commercial products which provide a standard GSS-API interface. The CertificateHandling object may interact with external services for certificate and CRL distribution, for example offered in conjunction with a CA TTP. Use of the LDAP protocol (RFC 1777, 1995) is envisaged for fetching of certificates and CRLs.

Note that in order to establish a secure management association, a management entity must have disabled automatic connection mechanisms, and take explicit control over the association.

\subsection{Adapter component}

The security defined in TRUMPET must be generic enough to be able to be used within various management environments, comprising different TMN platforms (HP OpenView, IBM Netview 6000 , etc.) and interfaces to the OSI stack. Therefore, there is a need to translate the specific data structures (syntax) of the MAE to a form that can be handled by the generic SMASC. This is the task of the Adapter component, which contains an Adapter object, and possibly further objects for encoding / decoding, as discussed in Section 5.3.

The MAE's specific data structures are protocol entities such as CMIS requests or XOM objects. There must be one adapter object per protocol, and extensibility of TRUMPET's security package to new environments is possible by coding new adapter objects.

The MAE must explicitly call the corresponding Adapter object to protect all Protocol Data Units (PDU) to be sent to the peer MAE. An Adapter object must have detailed knowledge about the protocol it is related to, as it must perform the following tasks on each PDU :

$\lambda$ extract the type of operation (like get / set / action);

$\lambda$ extract data which can be relevant for access control (i.e. target MIB object);

$\lambda$ extract the data fields that are concerned with protection (integrity and / or confidentiality);

$\lambda$ format the extracted information in the neutral representation of the SMASC;

$\lambda$ call the $S M A S C$ interface to verify access control and protect the required fields (integrity, confidentiality, non-repudiation);

$\lambda$ reformat the output of the SMASC's protect call into a valid PDU for the specific protocol. The protected PDU can then be sent by means of the ordinary communication stack to the peer entity. This entity performs the reverse operations on each received PDU to perform access control (for all operations) and check the protection (integrity and confidentiality).

\subsection{Secure management association establishment}

Figure 4 shows the event trace diagram for the establishment of a secure association from the initiator side. The security context is established at the same time as the establishment of the association. Security context establishment requires one or two message exchanges with the target MAE, depending on the type of authentication mechanism used. The association 
scenario shown corresponds to a two-way security context negotiation. Negotiation using more than two exchanges cannot be performed using the ACSE protocol.

Operations 4, 5 and 9 are security calls as specified by the GSS-API programming interface. GSS_Acquire_cred() establishes the authentication information of the initiator MAE. GSS_Init_sec_context() returns a security token that is transferred to the target MAE using the ACSE authentication field. The target MAE gives this security token as input to GSS_Accept_sec_context(). For a two-way security context establishment, this function returns another security token that is returned to the initiator MAE for validation.

In step 6, the Assoc_req operation stands for a number of operations on the communication API of the management platform, denoted by a ComProvider component.

Operation 10 is an auditing procedure that records operations that are relevant to security. For simplicity, there is only one audit call during the security context establishment procedure. Note that this call should also be used to log error messages from operations 1-9. Other management operations, such as the successful completion of the association establishment procedure, should also be recorded by the MAE.

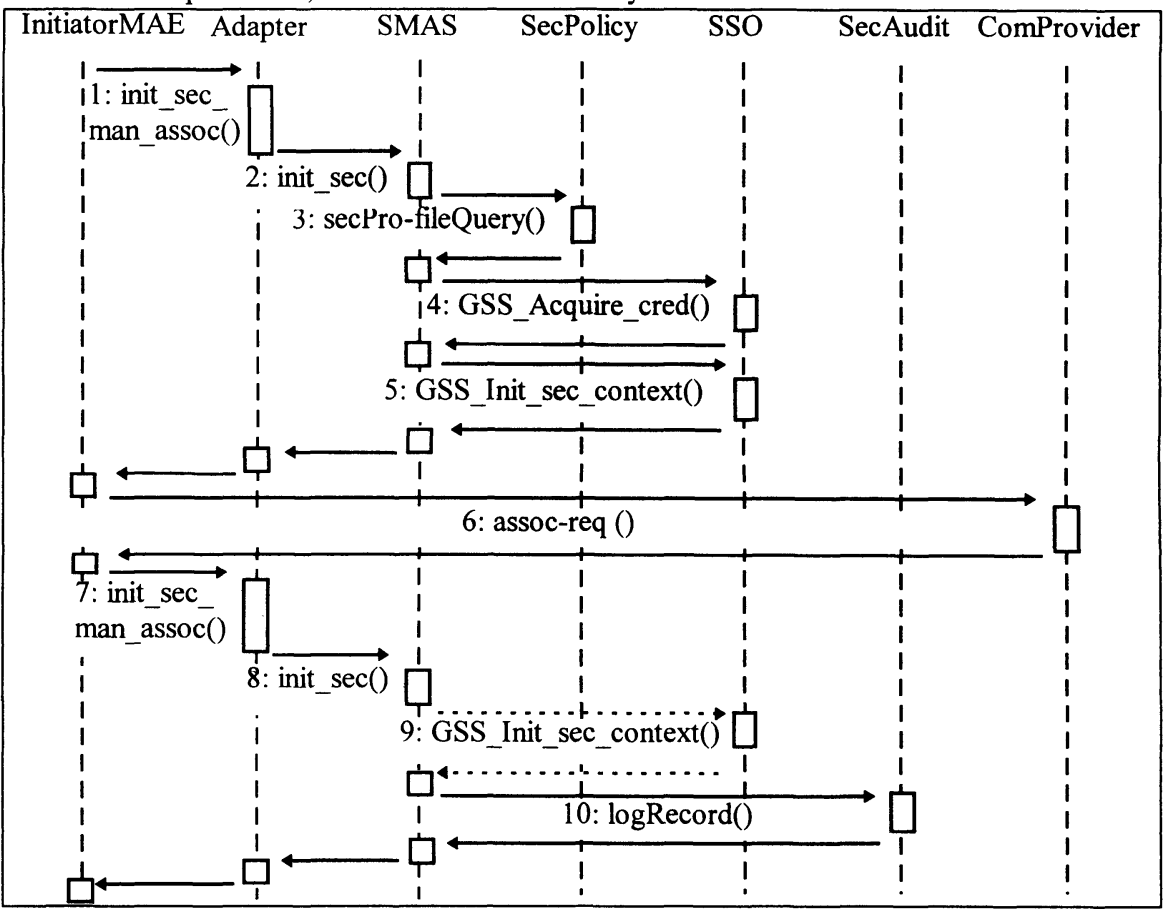

Figure 4 ETD of the SMAS on the initiator side. 


\section{SECURE MANAGEMENT OPERATIONS}

\subsection{Decomposition of access control into components}

The Access Control service is first decomposed into components which again are described by their respective object classes. The main functionalities are the Access control Enforcement Function (AEF), the Access control Decision Function (ADF) and the $a c M I B$ containing the management information (authorisations) needed for access control. The AEF is build into the $S M A S C$ which already has the needed contracts to the MAE. When access control is required, the $S M A S C$ uses the ControlAccess contract to interact with the AccessDecision component. The AccessDecision component will use the AccessacMIB contract to interact with the acMIB. Security Audit of access control shall be enforced by the AEF. The AEF is integrated in the SMASC which already provides the contract SecEventReport to the Security Audit component. This is shown in Figure 5.

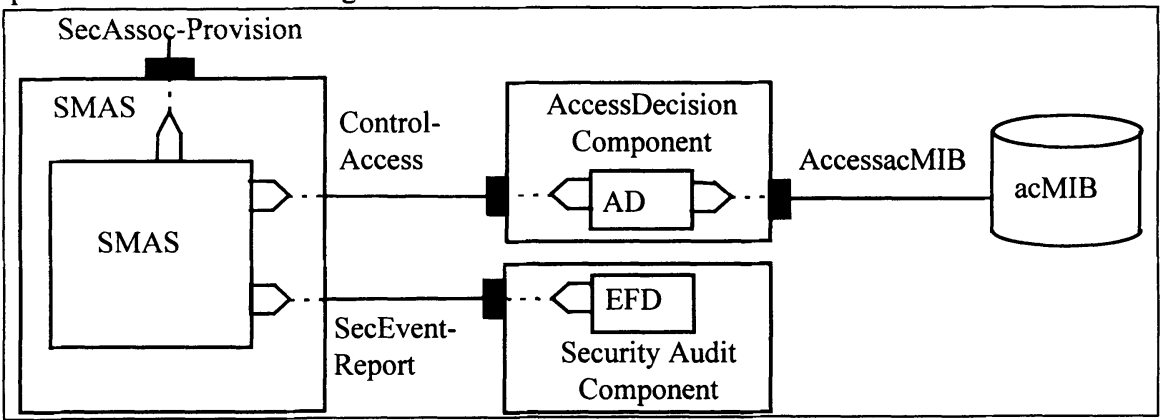

Figure 5 Decomposition of the access control service into components.

\subsection{Integrity and confidentiality of per-message management operations}

On commercial platforms, integrity and confidentiality must be implemented above CMISE, since the internals of the protocols stack are inaccessible. This has several drawbacks :

$\lambda$ the security services become much more application dependent.

$\lambda$ it requires extra encoding / decoding of application data to / from an application independent format - this is normally the task of the presentation layer in the protocol stack;

$\lambda$ the security transformations may have to be applied to several fields for a single CMISE service request - optimisation of the security transformations for several fields increases the complexity of the algorithms;

$\lambda$ for confidentiality, it requires a modification of application data types.

Figure 6 shows the procedure for integrity and confidentiality protection for sending data. We assume that a security context has been established and that it is accessible through the SSO to perform the security transformations. Although the integrity and confidentiality services are shown together, they only share the encoding/ decoding part. They can be activated/ deactivated without effecting the processing related to the other security services. 
The application data corresponds to a set of parameters for a CMIS service request or response. The parameter values are encoded using an ASN.1 encoder. For integrity, the encoded parameters are concatenated, possibly adding further information, like a time stamp or a sequence number, before generation of the Integrity Check Value (ICV). It is also be possible to perform selected field integrity, but in this case there must be some extra information contained in the application data (parameters) to determine which parameters to use for ICV verification. Because integrity is performed separately from confidentiality, the ICV is generated by a cryptographic algorithm.

The ICV may then be concatenated with Access Control Information (ACI), and possibly other information. The result is transferred to the peer entity using the CMIS access control field. Note that there is no access control field for event reports. If the (optional) event information parameter of event reports is supported, the ICV and other information could be transferred using this field.

For confidentiality, each encoded parameter is padded (if necessary) and encrypted separately (possibly sharing encryption algorithm information) and then transferred using the appropriate CMIS field. The parameters are not concatenated before encryption because the result would have to be put into a single parameter. We do not consider it reasonable to transfer this result in the CMIS access control field and put dummy values in the parameters.

Encryption is illustrated using dotted lines because it can only be performed on CMIS parameters which are able to accept a data type suitable for an encrypted value. For example, the Invoke Identifier parameter cannot accommodate an encrypted value. If a particular parameter requires confidentiality and the corresponding CMIS parameter data type is not suitable, it must be added to the access control field, which is not regarded as acceptable.

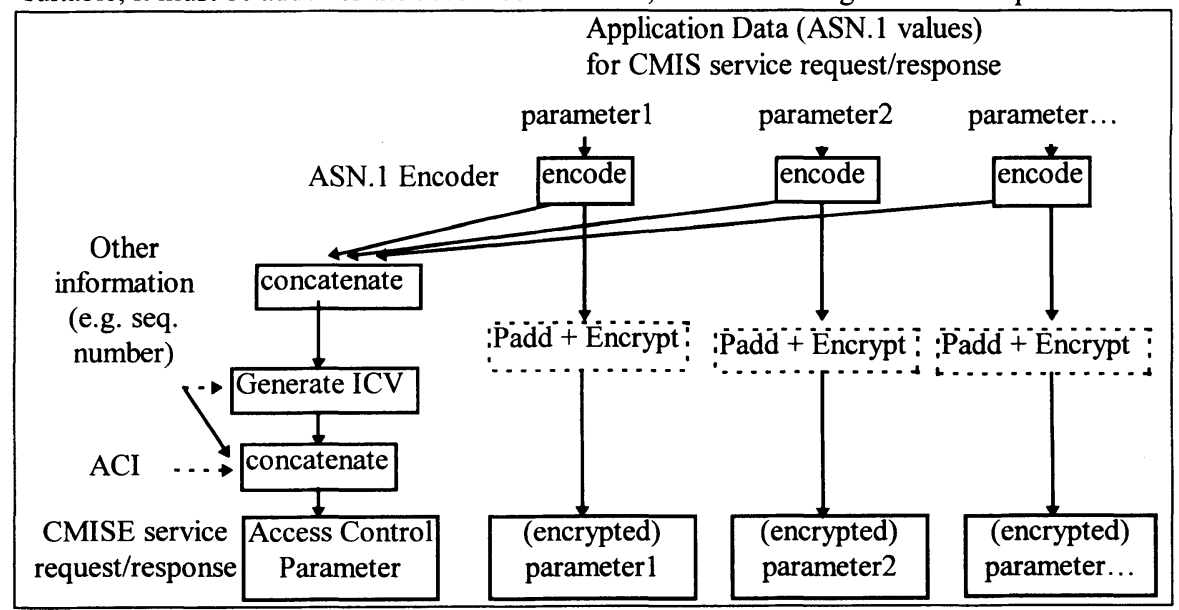

Figure 6 Integrity and confidentiality architecture on the sender side. 


\subsection{Components for integrity and confidentiality protection}

The integrity and confidentiality related cryptographic operations are implemented as operations on the contractual interface of the SMAS object. This is necessary because such operations need access to critical security parameters (i.e. security context, selected algorithms) initiated during the secure managament association establishment. The cryptographic operations are performed by the SSO object by calls over its GSS-API interface.

Encoding / decoding is necessary, for encoding of the application data in a machine and implementation language independent way, more specifically a byte string on which the $S M A S$ can work. For CMIS operations, this encoding must be carried out by ASN.1 encoding / decoding resulting in a universal (contiguous) BER representation (ITU-T X.683). The ASN.1 definitions of the CMIS service requests / responses are parsed by a compiler. This compiler generates code stubs that describe equivalent data structures and implements encode and decode functions. The generated code is used to build the ASN.1 Encoder object, which is included in the Adapter component. The impact of this procedure on the communication speed remains to be studied, but it is clear that simpler encoding methods are insufficient for complex structures like managed objects.

The integrity and confidentiality mechanisms are reflected through the use of the SE_CMISE portable, secured data type, which is derived from the equivalent non-secured CMISE request / response type. The SE_CMISE is sent to the target management application entity using the XOM / XMP-API interfaces of the ComProvider object.

No particular symmetric encryption algorithm has been mandated by TRUMPET's security policies. Since all processing related to symmetric encryption and decryption, including key management, is done by the SSO object, behind the GSS-API, a change of algorithm has no impact on the architecture, nor on other components or objects. TRUMPET bases its implementation on available software encryption packages, which typically offers a choice between DES, triple-DES, or IDEA (SECUDE, 1997).

\subsection{Procedures for per-message protection}

The necessary interactions between objects for protection of outgoing operation requests are illustrated in the ETD of Figure 7. If access control is required (optional on the initiating side, mandatory on the receiving side), the SMAS object will ask for this. The acDecision object will interact with the $a c M I B$ (not shown) to get the correct information. The SMAS object will enforce the necessary action (grant, deny, notification - logRecord()). 


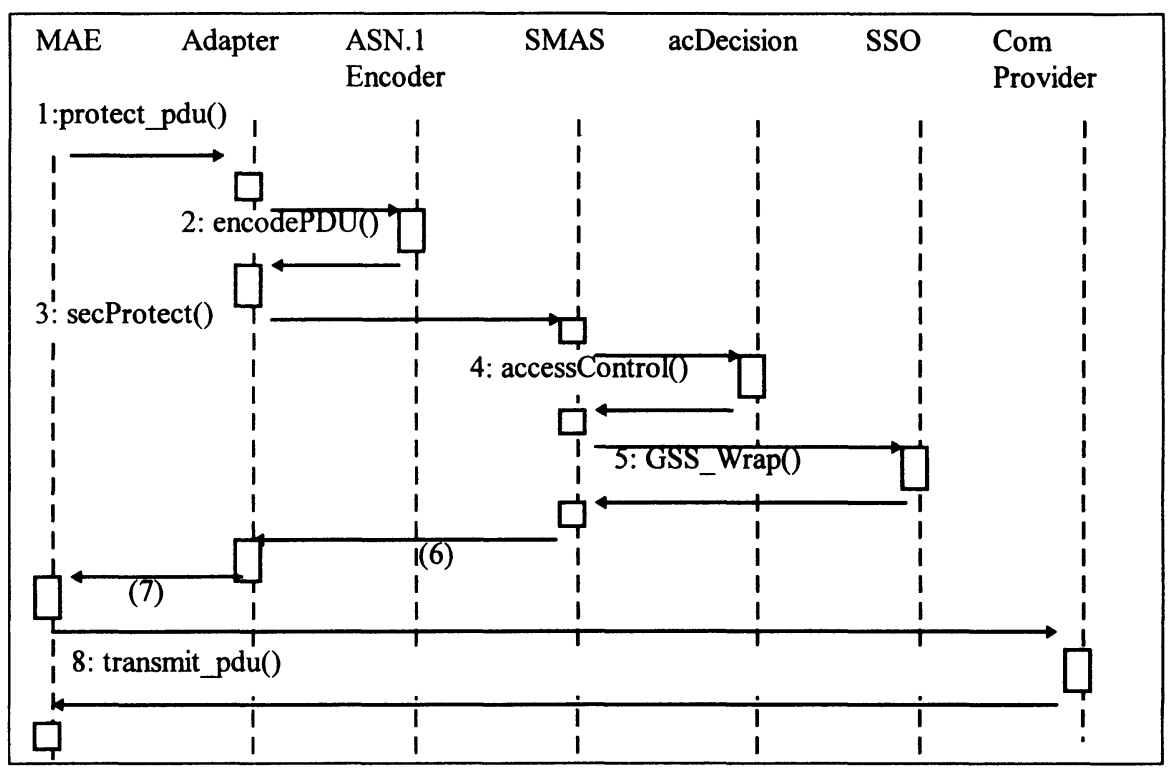

Figure 7 ETD of per-message protection operations on the sending side.

Figure 7 also shows the protection (integrity and / or confidentiality of all or selected data, depending on the domain's security policy) of a $M A E$ object (PDU).

The protect_pdu() call has to be inserted in the MAE code before each call to the communication stack in order to transmit a PDU to the peer entity. The syntax is typically that of a protocol entity (CMIP request or XOM object), and the call is made to an adapter object.

The adapter object extracts information necessary for access control, and calls the ASN.1 Encoder object to translates the protocol entity into a byte-code representation (architecture neutral) which can then be processed by the SMAS. In this case, the byte code will be BER. In other cases, it may be a different representation that may be handled as a byte string.

Operation 6 represents the output from the $S M A S$ 's protection of the byte-code object. The adapter then repackages the protected data in the $M A E$ 's syntax, and the result is returned to the application in operation 7. The $M A E$ can then transmit the protected PDU to the peer entity through calls to the local communication stack in operation 8 .

On the receiving side the received PDU is "unprotected", i.e. the integrity protection is verified, and confidentiality protection is decrypted. The PDU itself, together with the security context held by the $S S O$, contain enough information to identify the operations necessary. Thus, it is not necessary to involve the SecPolicy component on the receiving side. After "unprotection", access control is performed, and an audit call is done to record the results of both the integrity verification, decryption and access control. 


\section{NON-REPUDIATION AND SECURITY MANAGEMENT}

With respect to audit and alarm components, this paper only shows the contract towards the component responsible for receiving events. TRUMPET has developed an architecture for processing these events into log records and possibly alarms. There will be two different logs: one for ordinary events (message sent or received etc.), and another for security events (authentication succeeded / failed etc.). A tool for audit analysis will also be developed.

Generation of proofs suitable for non-repudiation of origin can easily be achieved in the current solution by using a public key algorithm for signing instead of a symmetric algorithm for integrity protection (with a mandatory time stamp). Later verification of a proof requires that messages are logged together with their signature. The audit analysis tool may then be enhanced to handle signature verification. The feasibility of non-repudiation at the granularity of individual management messages remains to be studied.

Non-repudiation of delivery would require a signed acknowledgement returned to the initiator. TRUMPET has at present not studied this service.

Management of symmetric keys is performed as an integral part of security context establishment. Authentication based on public key cryptography, in an inter-domain context, requires use of certificates issued by trusted CAs. TRUMPET assumes one CA within each domain, where these CAs in turn are certified under a public key infrastructure. The ICE-TEL project (ICE-TEL, 1997) is a suitable candidate for co-operation on this task.

In addition to key management, the main issue for security management is management of access rights (authorisation). TRUMPET will to some extent study this question, although it is an internal matter to the individual management domains.

\section{CONCLUSION}

This article has presented a practical way to add security features to TMN management systems based on currently available management platforms. In such platforms, which do not implement OSI security standards and are closed with respect to modifications to the protocol stack, all security services have to be implemented above the application layer.

\section{REFERENCES}

ACTS112-D2 (1996) TRUMPET: Inter-domain management with integrity - Deliverable 2B : Inter-TMN Security Policies.

ACTS112-D7 (1997) TRUMPET: Inter-domain management with integrity - Deliverable 7 : Security Policies \& System Architecture Specification.

CORBA (1994) X/Open and OMG : The Common Object Request Broker : Architecture and Specification.

GSS V2 (1996) IETF draft : Generic Security Service Application Program Interface, Version 2.

ICE-TEL (1997) http://www.darmstadt.gmd.de/ice-tel 
ITU-T M.3010 (1995) ITU-T M.3010 : Principles of a Telecommunications Management Network.

ITU-T X.274 (1993) ISO/IEC 10736 | ITU-T X.274 : OSI - Transport Layer Security Protocol.

ITU-T X.683 (1995) ISO/IEC 8824-4 | ITU-T X.683 : Information Technology - Abstract Syntax Notation One (ASN.1) - Parameterization of ASN.1 Specifications.

ITU-T X.830-833 (1995) ISO/IEC 11586 | ITU-T X.830-833 : OSI - Generic Upper Layers Security - Parts 1-4.

RFC 1508 (1993) J. Linn : Generic Security Service Application Program Interface.

RFC 1777 (1995) Lightweight Directory Access Protocol.

Rumbaugh et al. (1991) Object-oriented Modeling and Design, Prentice-Hall.

SECUDE (1997) http://www.darmstadt.gmd.de/secude

XMP (1992) X/Open Company Limited: XMP, Systems Management: Management Protocols API.

XOM (1991) X/Open Company Limited \& X.400 API Association : XOM, OSI-AbstractData Manipulation API, CAE Specification. 